\title{
Silent hepatic portal venous gas following upper gastrointestinal endoscopy
}

We present a case of benign hepatic portal venous gas (HPVG) following upper gastrointestinal endoscopy.

A 76-year-old man presented with epigastric pain and vomiting of coffeeground material. There was no remarkable medical history. Physical examination revealed mild epigastric tenderness. A plain film ( Fig. 1) showed gallbladder stones and localized ileus over the midabdomen. Upper gastrointestinal endoscopy revealed a gastric ulcer with gastric outlet obstruction. The patient denied any discomfort of the abdomen after the endoscopy. An abdominal computed tomography (CT) scan ( $\bullet$ Fig. 2) taken about 5 hours after the upper gastrointestinal endoscopy showed a contracted gallbladder with stones and a distended stomach with an arborized air collection in the portal system, distributed mainly in the peripheral region. However, there was no evidence of bowel ischemia or necrosis, and the patient underwent only conservative management. A follow-up abdominal CT scan ( $\bullet$ Fig. 3 ) 2 days later demonstrated no residual HPVG.

The finding of HPVG was first reported in 1955 [1]. Most cases of HPVG are related to mesenteric ischemia associated with bowel necrosis [2] and require an operation. However, HPVG can occur in several conditions that may be managed with conservative treatment only, such as gastric ulcers and endoscopic sphincterotomy [3]. Although the mucosal injury may be caused by serious pathology such as bowel ischemia, HPVG can occur in benign conditions such as acute gastric dilatation, iatrogenic bowel dilatation (e.g., following colonoscopy, endoscopic sphincterotomy), and abdominal trauma [3-5].

Our patient's asymptomatic HPVG may have been caused by the mucosal disruption related to the gastric ulcer and aggravated by the increased luminal pressure during endoscopy. The high intraluminal pressure during the endoscopic procedure allows the intraluminal gas to diffuse through damaged, or even undamaged bowel wall, and enter the venous circulation [5].

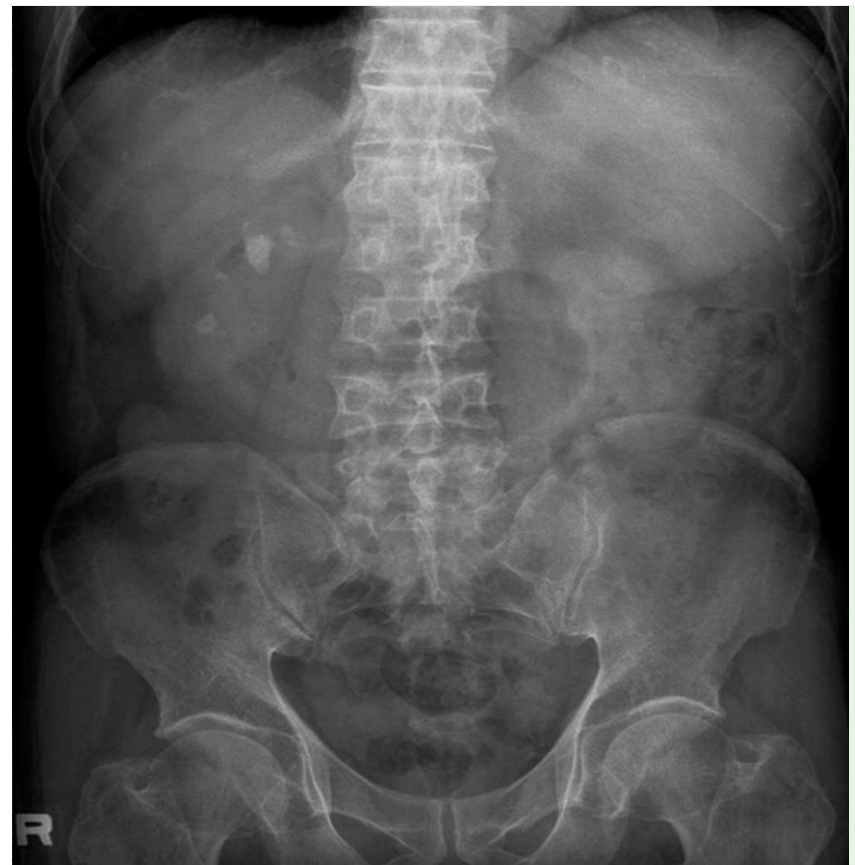

Fig. 1 Plain film showing gallbladder stones and localized ileus over the mid-abdomen.

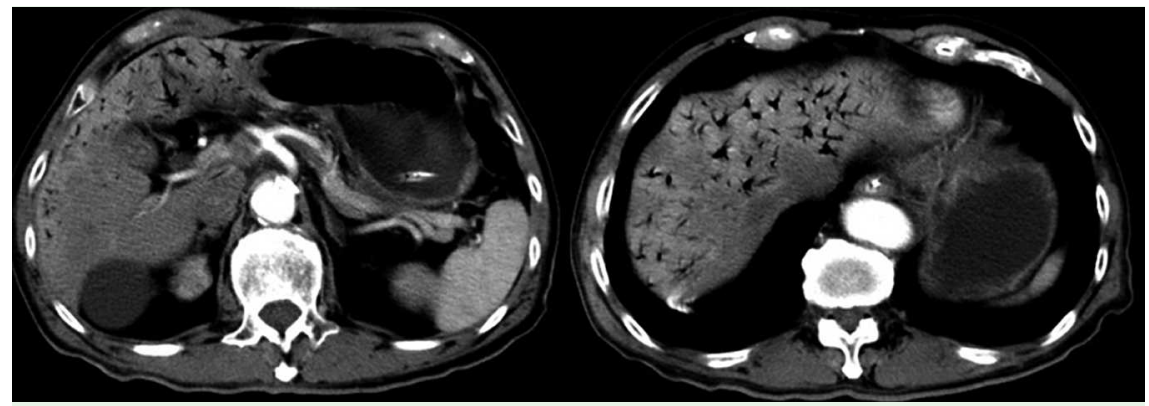

Fig. 2 Abdominal computed tomography (CT) scan showing a distended stomach with arborizing air collection in the portal system (mainly over the peripheral region).

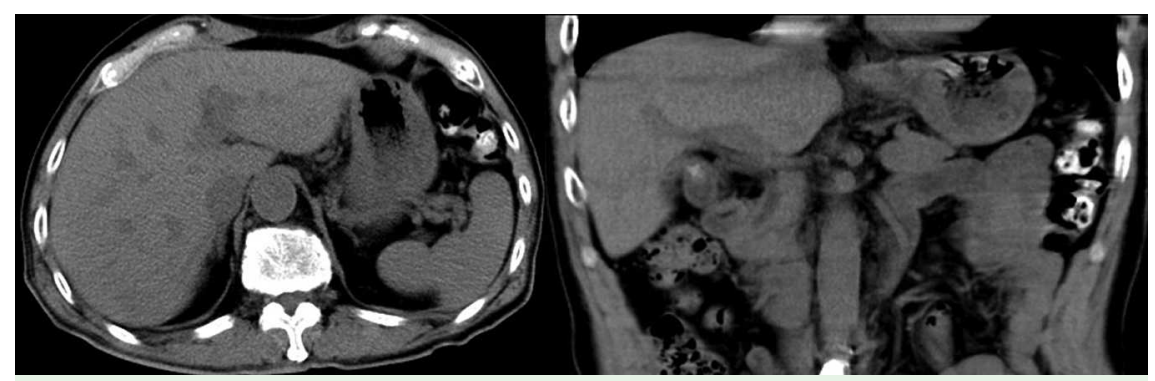

Fig. 3 Abdominal computed tomography (CT) scan showing no air in the portal system.

Asymptomatic HPVG can occur after upEndoscopy_UCTN_Code_CPL_1AH_2AB per gastrointestinal endoscopy and may be successfully managed with only conservative treatment. 
S. M. Kuo ${ }^{1}$, W. K. Chang ${ }^{2}$, C. Y. Yu , C. B. Hsieh ${ }^{1}$

${ }^{1}$ Division of General Surgery, Department of Surgery, Tri-Service General Hospital, National Defense Medical Center, Taipei, Taiwan

2 Division of Gastroenterology, Department of Internal Medicine, Tri-Service General Hospital, National Defense Medical Center, Taipei, Taiwan

${ }^{3}$ Department of Radiology, Tri-Service General Hospital, National Defense Medical Center, Taipei, Taiwan

\section{References}

1 Wolfe JN, Evans WA. Gas in the portal veins of the liver in infants: a roentgenographic demonstration with postmortem anatomical correlation. AJR Am J Roentgenol 1955; 74: $486-489$

2 Liebman PR, Patten MT, Manny J et al. Hepatic portal venous gas in adults: etiology, pathophysiology, and clinical significance. Ann Surg 1978; 187: 281 - 287

3 Herman JB, Levine MS, Long WB. Portal venous gas as a complication of ERCP and endoscopic sphincterotomy. Am J Gastroenterol 1995; 90: 828-829

4 Schindera ST, Triller J, Vock P et al. Detection of hepatic portal venous gas: its clinical impact and outcome. 2006; 12: $164-170$

5 Bani-Hani KE, Heis HA. Iatrogenic gastric dilatation: a rare and transient cause of hepatic-portal venous gas. Yonsei Med J 2008; 49: $669-671$
Bibliography

DOI 10.1055/s-0029-1214657

Endoscopy 2009; 41: E121-E122

(c) Georg Thieme Verlag KG Stuttgart · New York . ISSN 0013-726X

\section{Corresponding author}

\section{B. Hsieh, MD}

Division of General Surgery

Department of Surgery

Tri-Service General Hospital

National Defense Medical Center

No. 325 Cheng-gong Road (Sec. 2)

Neihu District 114

Taipei

Taiwan

Fax: +886-2-8792-7379

doc20443@yahoo.com.tw 